\title{
The human body as an energetic hybrid? New perspectives for chronic disease treatment?
}

\author{
Michał Gajewski ${ }^{1}$, Przemysław Rzodkiewicz ${ }^{2}$, Sławomir Maśliński ${ }^{3}$ \\ ${ }^{1}$ Department of Biochemistry and Molecular Biology, National Institute of Geriatrics, Rheumatology and Rehabilitation, Warsaw, Poland \\ 2Department of Gerontology and Public Health, National Institute of Geriatrics, Rheumatology and Rehabilitation, Warsaw, Poland \\ ${ }^{3}$ Department of General and Experimental Pathology, CEPT Laboratory, Medical University of Warsaw, Poland
}

\begin{abstract}
Inflammatory response is accompanied by changes in cellular energy metabolism. Proinflammatory mediators like plasma C-reactive protein, IL-6, plasminogen activator inhibitor-1, TNF- $\alpha$ or monocyte chemoattractant protein-1 released in the site of inflammation activates immune cells and increase energy consumption. Increased demand for energy creates local hypoxia and lead in consequence to mitochondrial dysfunction. Metabolism of cells is switched to anaerobic glycolysis. Mitochondria continuously generate free radicals that what result in imbalance that causes oxidative stress, which results in oxidative damage. Chronic energy imbalance promotes oxidative stress, aging, and neurodegeneration and is associated with numerous disorders like Alzheimer's disease, multiple sclerosis, Parkinson's disease or Huntington's disease. It is also believed that oxidative stress and the formation of free radicals play an important role in the pathogenesis of rheumatoid diseases including especially rheumatoid arthritis. Pharmacological control of energy metabolism disturbances may be valuable therapeutic strategy of treatment of this disorders. In recent review we sum up knowledge related to energy disturbances and discuss phenomena such as zombies or hibernation which may indicate the potential targets for regulation of energy metabolism.
\end{abstract}

Key words: energetic hybrid, metabolism, autoinflammatory diseases.

\section{The redistribution of energy by chemicals in the vascular-neuro-endocrine-lymphatic network}

\section{Redistribution of energy in acute inflammation}

The inflammatory response is a huge phenomenon. Activation of the immune system is related to a $30 \%$ increase in demand for energy [1]. Each inflammatory response includes three stages that may be characterized by changes in cellular energy metabolism [2, 3]. The first stage of the response is characterized by the mobilization of immune cells and their infiltration into the site of inflammation. Inflammatory mediators damage the endothelium causing increased fluid filtration and the formation of edema. The occurring edema leads to hypoxia. The hypoxic state forces cell metabolism adaptation. In hypoxic tissue, oxidative metabolism is only partially delayed. Processes such as the decomposition of adenosine triphosphate [ATP] to adenosine and the conversion of xanthine dehydrogenase into oxidase cause that the oxygen reaching the cell is not entirely reduced and reactive oxygen intermediates ( $\mathrm{ROI}$ ) are produced, i.e. oxygen atoms with additional unpaired electrons. That phenomenon is called the "oxygen paradox" [3]. The next stage is related to the revascularization process. Re-perfused tissue attracts a high number of neutrophils, which are the first-line defense of the immune system. The metabolism of these cells is based on anaerobic glycolysis, while the primary mechanism of their toxic effects is the ability to convert oxygen into

Address for correspondence:

Michał Gajewski, Department of Biochemistry and Molecular Biology, National Institute of Geriatrics, Rheumatology and Rehabilitation, Spartanska 1, 02-637 Warsaw, Poland, e-mail: michal.gajewski@spartanska.pl

Submitted: 29.11.2016; Accepted: 19.04.2017 
ROI. These two parallel processes - the "oxygen paradox" and production of ROI by neutrophils - result in the impairment of energy metabolism in the area of the ongoing inflammatory process. These first two stages of inflammation can be called stages of constant disturbance of oxygen metabolism $[3,4]$. The third stage is related to the resolution of inflammation. The metabolism switch of neutrophils is an essential element of the course of inflammatory response and can be regulated by several pharmacological treatments. In our experiments, we showed that during chemical-induced hypoxia, when the cellular level of ATP has decreased, the responsiveness of myocytes to muscarinic cholinergic (part of parasympathetic nervous system [PSNS]) stimulation increases significantly [5]. This increase in physiological response to muscarinic receptor stimulation was associated with an increase of muscarinic receptor expression (630\%) [5, 6]. Different subtypes of muscarinic receptors are expressed on neutrophils present in control and inflamed tissue. In our research, we demonstrated the presence of M3, M4, M5 muscarinic receptor subtypes in human blood neutrophils and the lack of mRNA for M4 muscarinic receptor subtype in human neutrophils isolated from the inflamed tissue of patients with rheumatoid arthritis (RA). The differential expression of muscarinic receptors from healthy and RA patients may contribute to an opposite response to cholinergic stimuli observed in neutrophils isolated from peripheral blood and inflamed tissue [7]. It's commonly accepted that adrenaline and other $\beta$-adrenergic receptor agonists of the sympathetic nervous system (SNS) decrease neutrophil activation are [8]. Contrary to that, the cholinergic agonist, increases the activity of neutrophils isolated from blood but decreases the activity of neutrophils isolated from the inflamed environment [8]. The opposite effects of $\beta$-adrenergic and muscarinic receptor agonists on the activity of neutrophils may reflect their opposing influences on inflammation, and may support the idea of both a "pro-inflammatory" and an "anti-inflammatory" effect of the PSNS [8].

Our results led us to an interesting finding: PSNS neurotransmitters may, similarly to SNS neurotransmitters, potently decrease inflammatory activity [9]. It can be suggested that PSNS, in acute inflammation in ischemic tissue, both decreasing myocyte contractility and neutrophil activity, provided a significant reduction in energy utilization and consequent tissue destruction.

\section{Redistribution of energy in chronic inflammation}

The transition from acute to chronic inflammation is a complex process that is not fully understood. One of the hypotheses defines it as the point of total con- sumption of available energy resources [2]. The amount of energy resources accumulated in the body of mammals is relatively constant and sufficient to meet the body's energy demands for about four weeks. At the end of the $19^{\text {th }}$ century, energy resources accumulated in the body of typical women and men held out for 28.3 and 25.3 days, respectively. The $20^{\text {th }}$ century brought changes in the lifestyle of humans that brought a significant increase of energy resources accumulated in the body. Recently, level of energy resources within the human body is sufficient for 43 days for women and 41 days for men [2]. In warm-blooded animals, most of the energy produced within the body is used to control constant body temperature. The progress of civilization has enabled us to control ambient temperature so actually, this does not apply to humans. We spend most of our life in a temperature that is close to the thermic neutral point $\left(23^{\circ} \mathrm{C}\right)$ at which the body does not require any energy to keep the temperature stable. Thus, the sign of our times, obesity results not only from energy consumed with food but mainly from the energy of the contemporary, much-changed environment [10]. Finally, modern humans primarily spend their energy resources on nervous and immune system activity. Moreover, the inflammatory process can be associated with the widely understood psychophysiological stress of modern society, which is surprisingly affected by biological, social, behavioral or religious factors. Finally, chronic inflammation is called "metabolic syndrome", and modern humans are still often called "inflammacitizens" [11]. Changes in energy production and flow are critical homeostatic regulators of physiological processes. Due to their functions, factors that regulate energy metabolism can be divided into elements providing high-energy substrates to energy-storing organs (primarily PSNS) and those providing such substrates to energy-consuming organs (especially SNS). The circadian rhythm sets the energy flow between energy consuming organs (brain, muscles, and immune system) and energy suppliers (liver, adipose tissue). During the day, energy is delivered mainly to the brain and muscles while at night to the immune system $[12,13]$. The third stage of inflammation is called the repair stage; but in chronic inflammation, normal oxygen metabolism does not begin. The reason is that cells cannot draw energy "locally" from the already destroyed extracellular cellular matrix (ECM), while the "systemic" energy sources have run empty due to continued over activation of the SNS. Most immune cells infiltrating inflammation affected tissues switch their metabolism. Survival of these cells within the inflammation site may influence the course of inflammation excitation or fixation $[12,13]$. The basic metabolism of neutrophils is based on anaerobic glycolysis, while non-activated 
lymphocytes produce energy primarily by oxidative phosphorylation. Hypoxia induces an oxidative burst of neutrophils and switch lymphocytes to anaerobic glycolysis [14]. Also, macrophages play a twofold role in inflammation. Non-activated macrophages derive energy from oxidative phosphorylation. On the one hand, macrophages activated in the inflammation site convert to "pro-inflammatory" macrophages of M1 type, which produce energy in the anaerobic glycolysis process. On the other hand, during the resolution of inflammation, macrophages of $\mathrm{M} 2$ type, with anti-inflammatory properties and repair functions, return to oxygen-dependent energy metabolism. It was found that in chronic inflammation, the major subtype of macrophages present in the site of inflammation is the M1 subtype [15]. Taken together, in chronic inflammation, the balance between energy storage and energy consumption processes is significantly disturbed. It seems that chronic inflammation can be defined as an adaptation to continued redistribution of energy, from energy-storing organs (primarily PSNS) to the activated immune system. In chronic inflammation, dislocation of high-energy substrates to energy-consuming organs (especially SNS) is notably disturbed. Taken together it can be suggested that decrease of PSNS activity may be responsible for chronic disease development.

\section{The neurodegeneration phenomena as a chronic inflammation process depend on energy disturbances}

Chronic neuroinflammation has been associated with many neurodegenerative disorders of the central nervous system, such as Alzheimer's disease (AD), multiple sclerosis, Parkinson's disease (PD), or Huntington's disease [16]. The key pathogenic role of oxidative stress has been suggested both in AD as well as PD. Langston et al. [17] described the occurrence of severe symptoms similar to PD in narcotic drug addicts to MMP+ (1-methyl4-phenylpyridinium), a neurotoxin that can enter the neurons. It was shown that MPP+ induces neurotoxicity primarily by blocking mitochondrial complex I activity, leading to ATP depletion and increased oxidative stress $[16,17]$. This observation links oxidative stress with the development of neuronal death and neural dysfunction $[16,17]$. Notably, energy disturbances, and in consequence mitochondrial dysfunction, is also a prominent feature in these diseases. Free radicals are made continuously by mitochondria and must be balanced by antioxidant defense to maintain redox homeostasis. The imbalance between harmful ROI and antioxidant defenses causes oxidative stress, which results in oxidative damage $[18,19]$. Consequently, dysfunction of the mitochondria seems to promote oxidative stress, aging, and neurodegeneration. In fact, mitochondrial dysfunction and oxidative stress, in general terms defined as an energy imbalance, constitute the most prominent features found in neurodegenerative phenomena such as Alzheimer's (AD) or Parkinson's disease (PD) [16-19].

\section{Extremal decrease of energy availability: a "zombie" phenomenon}

A substance called 'zombie powder' that contains tetrodotoxin, hyoscyamine or atropine, administered in an adequate dosage, due to anticholinergic activity, would reduce a victim's metabolism to the point that could be mistaken with death. However, the victim would remain alive and could be revived with the administration of an antidote [20, 21]. It was reported that patients with particularly severe and life-threatening tetrodotoxin (TTX) poisoning can be successfully treated with a cholinesterase inhibitor to a complete and uneventful recovery $[20,21]$. TTX toxicity is caused by the competitive and reversible block at the motor endplate as well as motor axon and muscle membrane. Increasing the release of acetylcholine (ACh - naturally occurring PSNS agonist) at the neuromuscular junction by anticholinesterase drugs can reverse this block. Thus, neostigmine, which reversibly binds and inactivates acetylcholinesterase, can increase ACh availability [20].

Dysautonomia is frequently observed in tetradotoxication. Although the precise mechanism is not entirely understood, it was demonstrated that dysautonomia associated with TTX depends on a counterbalance between PSNS and SNS changes in both the central and peripheral compartments. Nevertheless, the PSNS crisis is usually predominant in tetradotoxication [21-23]. The blockade of neural ACh receptor with TTX or atropine, respectively, reduced the stimulatory effect on contractility. Moreover, it was shown that $\mathrm{H}_{2} \mathrm{~S}$, an important signaling molecule, has enhance ACh release from central preganglionic terminals [24, 25]. Interestingly, $\mathrm{H}_{2} \mathrm{~S}$ may exert effects that are related to neurodegenerative disorders such as Alzheimer's (AD) and Parkinson's disease (PD). A new hope in chronic inflammation as neurodegeneration treatment is to discover the role of $\mathrm{H}_{2} \mathrm{~S}$ in the energy-disturbed phenomenon [26].

\section{The possible molecular mechanism of transfer of energy metabolism in living organisms: the gasotransmitters}

Carbon monoxide (CO), nitric monoxide (NO), and hydrogen sulfide $\left(\mathrm{H}_{2} \mathrm{~S}\right)$, are gases that are generated endogenously $[27,28]$. Stimulation of ACh receptors on endothelial cells activates $\mathrm{Ca}^{2+}$-calmodulin (CaM), which 
in turn stimulates endothelial NO synthase (eNOS), cystathionine y-lyase (CSE), and $\mathrm{H}_{2} \mathrm{O}$ to produce $\mathrm{NO}, \mathrm{H}_{2} \mathrm{~S}$, and $\mathrm{CO}$, respectively. $\mathrm{NO}$ and $\mathrm{CO}$ can diffuse into the surrounding smooth muscle cells, where they activate soluble guanylyl cyclase (sGC) to produce cyclic GMP (cGMP) and affect the actin-myosin cross-bridge. $\mathrm{H}_{2} \mathrm{~S}$ can also diffuse into smooth muscles, where it activates $\mathrm{K}+$ channels. The actions of all three gases lead to vasorelaxation [27]. At physiological levels, each of these gases inhibits inflammation and has cytoprotective properties [27, 28]. $\mathrm{H}_{2} \mathrm{~S}$, NO, and $\mathrm{CO}$ are classic inhibitors of cytochrome $\mathrm{C}$ oxidase, the terminal and crucial enzyme of the electron transport chain in mitochondria [30]. $\mathrm{CO}, \mathrm{NO}$, and $\mathrm{H}_{2} \mathrm{~S}$ may suppress oxidative phosphorylation and decrease the intensity of energy production. It may be assumed that PSNS acts on energy metabolism indirectly via gasotransmitters $[27,28]$. Formerly, when there was no free oxygen $\left(\mathrm{O}_{2}\right)$ in the Earth's atmosphere and no ozone sphere $\left(\mathrm{O}_{3}\right)$, ultraviolet light arrived on Earth without obstacles and its intensity was 30 times greater than presently. The first source of oxygen in the atmosphere was not, as is commonly believed, the classical process of photosynthesis but its chemical equivalent in which $\mathrm{H}_{2} \mathrm{~S}$ was a reducer of $\mathrm{CO}_{2}$. Sulfide split water molecules into hydrogen, oxygen, and their derivatives (superoxide anions, hydrogen peroxide, hydroxyl radicals), with the release of energy [29]. $\mathrm{H}_{2} \mathrm{~S}$, the functional part of PSNS, is an old evolutionary remnant of the original nutrition and now is used for controlled intensity of aerobic respiration.

\section{Suspended hibernation: $\mathrm{H}_{2} \mathrm{~S}$ as a gasotransmitter and energy relocation example}

Hibernation is a state of the body in which metabolic processes are inhibited but do not result in termination. It was shown that the effect of "suspended animation" is likely related to the inhibition of mitochondrial electron transport mainly by a high local concentration of $\mathrm{H}_{2} \mathrm{~S}[30$, 31]. Exposing mice to a sub-toxic concentration of $\mathrm{H}_{2} \mathrm{~S}$ (20-80 ppm) dose-dependently reduced metabolism. Exposition to $\mathrm{H}_{2} \mathrm{~S}$ reduced $\mathrm{O}_{2}$ consumption by mice by 60-70\% within 5-10 min. A slow decline of metabolism to around $5 \%$ of basal levels was detected. This more gradual decline parallels the reduction in body temperature that follows exposure. Core body temperature steadily declines to around $2^{\circ} \mathrm{C}$ above ambient temperature over the course of several hours. After cessation of $\mathrm{H}_{2} \mathrm{~S}$ exposure, metabolism recovers to basal levels with no apparent toxic effects [32]. It was shown that hibernation of Scandinavian bears, similarly to mice, is associated with highly significant changes in plasma $\mathrm{H}_{2} \mathrm{~S}$ metabolites and enhanced intracellular reduced glutathione
(GSH) levels. [33]. Overall, total sulfide did not change significantly in either plasma or red blood cells (RBC) upon hibernation. These observations indicate that the generation and consumption of $\mathrm{H}_{2} \mathrm{~S}$ is balanced and the role of $\mathrm{H}_{2} \mathrm{~S}$ in hibernation is rather related to a shift in the way it is produced and consumed [33]. Low arterial $\mathrm{O}_{2}$ tension and high hemoglobin $\mathrm{O}_{2}$ affinity in hibernating bears provides conditions of low $\mathrm{O}_{2}$, under which $\mathrm{H}_{2} \mathrm{~S}$ can diffuse into surrounding cells and contribute to suppressing mitochondrial respiration. Similar $\mathrm{O}_{2}$-linked processes could also take place in cells and tissues other than blood. Suppression of $\mathrm{O}_{2}$ consumption in hibernation necessarily originates from the mitochondria, where $90 \%$ of whole-animal $\mathrm{O}_{2}$ consumption takes place. Other molecules with similar properties may also be involved in the metabolic suppression of hibernators [33]. It was observed that hibernating bears have a high tolerance of oxidative stress and increased regenerative capacity. The ubiquitous GSH is a fundamental element in the thiol-dependent cellular defense against ROS and redox imbalance. It was found that hibernation in free-ranging brown bears is associated with highly significant changes in plasma $\mathrm{H}_{2} \mathrm{~S}$ metabolites and enhanced intracellular GSH levels. Interestingly, mitochondrial respiration in the cardiac muscle and brain was not depressed in hibernating ground squirrels, suggesting that during winter hibernation energy is preferentially allocated to these two vital organs [34, 35].

In addition to inhibiting mitochondrial respiration, $\mathrm{H}_{2} \mathrm{~S}$ also has marked effects on circulation, by acting as a hypoxic vasoconstrictor or vasodilator in pulmonary and systemic circulation. In systemic circulation, an increase in peripheral resistance would maintain adequate blood pressure and prolong energy stores in the body by redistributing blood flow to the most demanding organs $[36,37]$. Conversely, in lung circulation, $\mathrm{H}_{2} \mathrm{~S}$ may contribute to hypoxic vasoconstriction thereby helping to maintain high oxygen saturation in arterial blood [36, 37]. Revsbech et al. [33] suggested these complementary abilities of $\mathrm{H}_{2} \mathrm{~S}$ and $\mathrm{NO}$ to induce controlled and reversible hypometabolic states. On the other hand, not only PSNS but also SNS may be involved in modulating of energy metabolism by gasotransmitters. As was shown [38] environmental signals may trigger the release of the hormone noradrenaline stimulating SNS receptors which activate eNOS, and subsequently lead to production of NO, and finally to modification of energy metabolism.

\section{Summary}

Controlling energy and control of metabolism disturbances may be valuable therapeutic strategy of treatment of disorders in which developing chronic inflammation, dislocation of high-energy substrates 
to energy-consuming organs and the immune system activation occur. Understanding the physiological processes occurring in hibernating organism, including the effects of gasotransmitters of PSNS and SNS on energy metabolism and circulation, will help in improving new therapeutic applications of energy relocation in future therapies of chronic diseases.

\section{The authors declare no conflict of interest.}

\section{References}

1. Straub RH. Evolutionary medicine and chronic inflammatory state-known and new concepts in pathophysiology. J Mol Med (Berl) 2012; 90: 523-534.

2. Romson JL. Reduction of the extent of ischemic myocardial injury by neutrophil depletion in the dog. Circulation 1983; 67: 1016-1023.

3. Aller MA, Arias JL, Arias J. Post-traumatic inflammatory response: perhaps a succession of phases with nutritional purpose. Med Hypotheses 2004; 63: 42-46.

4. Delmastro-Greenwood MM, Piganelli JD. Changing the energy of an immune response. Am J Clin Exp Immunol 2013; 2: 30-54.

5. Gajewski M, Laskowska-Bożek H, Moutiris JA, et al. Enhanced responsiveness of rat cardiac myocytes to muscarinic cholinergic stimulation during chemically-induced hypoxia. Acta Neurobiol Exp 1993; 53: 79-91.

6. Gajewski M, Laskowska-Bożek, Maśliński S, et al. Increase in the expression of muscarinic cholinergic receptors in isolated, neonatal rat cardiac myocytes treated with potassium cyanide. Acta Biochim Pol 1991; 38: 61-65.

7. Bany U, Gajewski M, Księżopolska-Pietrzak K, et al. Expression of mRNA encoding muscarinic receptor subtypes in neutrophils of patients with rheumatoid arthritis. Ann NY Acad Sci 1999; 876: 301-304

8. Gajewski M, Gujski M, Księżopolska-Pietrzak K, et al. The influence of adrenergic and cholinergic agents on the respiratory burst of human neutrophils from peripheral blood and synovial fluid. J Physiol Pharmacol 1997; 48: Suppl. 2: 72-79.

9. Gajewski M, Moutiris JA, Maslinski S, et al. The neuromodulation aspects of ischemic myocardium: the importance of cholinergic system. J Physiol Pharmacol 1995; 46: 107-125.

10. Hansen JC, Gilman AP, Odland JØ. Is thermogenesis a significant causal factor in preventing the "globesity" epidemic? Med Hypotheses 2010; 75: 250-256.

11. Aller MA, Arias N, Fuentes-Julian S, et al. Coupling inflammation with evo-devo. Med Hypothesis 2012; 78: 721-731.

12. Straub RH, Cutolo $M$, Buttgereit $F$, et al. Energy regulation and neuroendocrine-immune control in chronic inflammatory diseases. J Intern Med 2010; 267: 543-560.

13. Straub RH, Besedovsky HO. Integrated evolutionary, immunological, and neuroendocrine framework for the pathogenesis of chronic disabling inflammatory diseases. FASEB J 2003; 17: 2176-2183.

14. Brand KA, Hermfisse U. Aerobic glycolysis by proliferating cells: a protective strategy against oxygen species. FASEB I 1997; 11: 388-395.
15. Gajewski M, Rzodkiewicz P, Wojtecka-Łukasik E. The role of physiological elements in the future therapies of rheumatoid arthritis. II. The relevance of energy redistribution in the process of chronic inflammation. Reumatologia 2015; 53: 40-45.

16. Leszek J, Barreto GE, Gąsiorowski KK, et al. Inflammatory Mechanisms and Oxidative Stress as Key Factors Responsible for Progression of Neurodegeneration: Role of Brain Innate Immune System. CNS Neurol Disord Drug Targets 2016; 15: 329-336.

17. Langston JW, Ballard P, Tetrud JW, et al. Chronic Parkinsonism in humans due to a product of meperidine-analog synthesis. Science 1983; 219: 979-980.

18. Yun JW, Ahn JB, Kang BC. Modeling Parkinson's disease in the common marmoset (Callithrix jacchus): overview of models, methods, and animal care. Lab Anim Res 2015; 31: 155-165.

19. Yan MH, Wang X, Zhu X. Mitochondrial defects and oxidative stress in Alzheimer disease and Parkinson disease. Free Radical Biol Med 2013; 62: 90-101.

20. Albuquerque UP, Melo JG, Medeiros MF, et al. Natural products from ethnodirected studies: revisiting the ethnobiology of the zombie poison. Evidence-Based Complementary and Alternative Medicine vol. 2012, articles ID 202508, 19 pages doi:10.1155/2012/202508.

21. Littlewood R, Douyon C. Clinical findings in three cases of zombification. Lancet 1997; 350: 1094-1096.

22. Kheifets J, Rozhavsky B, Solomonovich ZG, et al. Severe tetrodotoxin poisoning after consumption Lagocephalus sceleratus (Pufferfish, Fugu) fished in Mediterranean Sea, treated with cholinesterase inhibitor. Case Rep Crit Care 2012; 2012: 782507. doi: 10.1155/2012/782507.

23. Yin HL, Lin HS, Huang CC, et al. Tetrodotoxication with Nassauris Glans: A possibility of tetrodotoxin spreading in marine products near Pratas Islands. Am J Trop Med Hyg 2005; 73 : 985-990.

24. Johar K, Priva A, Wong-Riley MT. Regulation of $\mathrm{Na}(+) / \mathrm{K}(+)$-ATPase by neuron-specific transcription factor Sp4: implication in the tight coupling of energy production, neuronal activity and energy consumption in neurons. Eur J Neurosci 2014; 39: 566-578.

25. Fernandes VS, Xin W, Petkov GV. Novel mechanism of hydrogen sulfide-induced guinea pig urinary bladder smooth muscle contraction: role of BK channels and cholinergic neurotransmission. Am J Cell Physiol 2015; 309: C107-C116.

26. Gong QH, Shi XR, Hong ZY, et al. A new hope for neurodegeneration: possible role of hydrogen sulfide. J Alzheimers Dis 2011; 24 (Suppl 2): 173-182.

27. Szabo C. Gaseotransmitters: New Frontiers for Translational Science. Sci Transl Med 2010; 2: 59ps54; doi:10.1126/scitranslmed.3000721.

28. Gajewski M, Maslinski S, Rzodkiewicz P, et al. Gas-Therapy in Rheumatoid Arthritis Treatment: When West Meets East - Actual Medical Concepts with Ancient World Ideas. Innovative Rheumatology InTech (Charpter 9) 2013; 189-213. Available at: https://www.intechopen.com/books/innovative-rheumatology/gas-therapy-in-rheumatoid-arthritis-treatment-whenwest-meets-east-actual-medical-concepts-with-anci.

29. Cloud P. Atmospheric cycles of carbon and sulfur and their effect on atmospheric oxygen over Phanerozoic time. Paleogeography, Paleoclimatology, Paleoecology 1988; 75: 97-122. 
30. Nichols P, Marshall DC, Cooper CE, et al. Sulfide inhibition of and metabolism by cytochrome c oxidase. Biochem Soc Transaction 2013; 41: 1312-1316.

31. Modis K, Bos EM, Calzia E, et al. Regulation of mitochondrial bioenergetics function by hydrogen sulfide. Part II. Pathophysiological and therapeutic aspects. Br J Pharmacol 2014; 171: 2123-2146.

32. Blackstone E, Morrison M, Roth MB. H2S induces a suspended animation-like state in mice. Science 2005; 308: 518.

33. Revsbech IG, Shen X, Chakravarti R, et al. Hydrogen sulfide and nitric oxide metabolites in the blood of free-8ranging brown bears and their potential roles in hibernation. Free Rad Biol Med 2014; 73: 349-357.

34. Chung DJ, Szyszka B, Brown JCL, et al. Changes in the mitochondrial phosphoproteome during mammalian hibernation. Physiol Genomics 2013; 45: 389-399.
35. Gallagher K, Staples JF. Metabolism of brain cortex and cardiac muscle mitochondria in hibernating 13-lined ground squirrels Ictidomys tridecemlineatus. Physiol Biochem Zool 2013; 86: 1-8.

36. Hochachka PW, Somero GN. Biochemical Adaptation: Mechanism and Process in Physiological Evolution. Oxford Univ Press, Oxford 2002.

37. Ramirez JM, Folkow LP, Blix AS. Hypoxia tolerance in mammals and birds: from the wilderness to the clinic. Ann Rev Physiol 2007; 69: 113-143.

38. Bossy-Wetzel E, Lipton SA. Nitric oxide signaling regulates mitochondrial number and function. Cell Death Differ 2003; 10: 757-760. 